\title{
The Three-Stage Model Based on Strain Strength Distribution for the Tensile Failure Process of Rock and Concrete Materials ${ }^{\star \star}$
}

\author{
Rukun Guo Shihai Li Dong Zhou* \\ (Institute of Mechanics, Chinese Academy of Sciences Beijing 100190, China)
}

Received 10 July 2015, revision received 31 August 2016

\begin{abstract}
A three-stage model is introduced to describe the tensile failure process of rock and concrete materials. Failure of the material is defined to contain three stages in the model, which include elastic deformation stage, body damage stage and localization damage stage. The failure mode change from uniform body damage to localization damage is expressed. The heterogeneity of material is described with strain strength distribution. The fracture factor and intact factor, defined as the distribution function of strain strength, are used to express the fracture state in the failure process. And the distributive parameters can be determined through the experimental stress-strain curve.
\end{abstract}

KEY WORDS three-stage constitutive model, tensile failure, distribution of strain strength, fracture factor, rock and concrete

\section{Introduction}

Rock and concrete materials are usually discontinuous with joints and pre-existing cracks on the micro scales ${ }^{[1]}$. The internal micro cracks and micro defects will occur and grow in the failure process of the brittle material ${ }^{[2]}$. It is difficult to describe the failure behavior of rock and concrete materials because of its discontinuity and heterogeneity. Tensile failure is one of the most common failure modes of rock and concrete materials. Commonly, the tensile strength is much lower than the compressive strength and shear strength of these materials. Although rock and concrete would hardly be in the simple state of pure tension or direct tension, the splitting failure in compression and the bending failure of rock and concrete beams are both caused by the tensile stress in local domain. So it is of great significance to understand and describe the constitutive relationship, the bearing capacity, the mechanism of deformation and the failure during tensile failure process ${ }^{[3]}$.

The constitutive relationship of materials is usually expressed by the relationship between stress and strain, which could be described intuitively with the complete stress-strain curves. The complete stress-strain curve of uniaxial tension test is usually divided into two parts by means of simple analysis of the experimental curve, e.g. the rising part before the peak stress is reached and the strain softening part after ${ }^{[4]}$. The fitting methods of simple linear type, polynomial type, fractional type and mixed type have been used to fit the complete stress-strain curves ${ }^{[5-7]}$. However, most of these methods are usually phenomenological without considering the mechanism of mesoscopic damage and fracture.

* Corresponding author. E-mail: zhoudong@imech.ac.cn

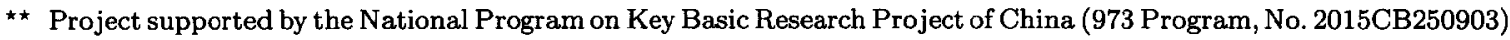
and CAS Strategic Priority Research Program (B) (No. XDB10030303). 
The damage and fracture will occur before the peak stress is reached in the tensile failure process, which is the internal reason for the nonlinear phenomenon on the macroscopic stress-strain curves. The research results in mesoscopic scale can explain the damage mechanism for tensile failure process of rock. And some damage constitutive models considering mesoscopic mechanical properties have been proposed ${ }^{[8,9]}$. However, these models are homogeneous models without considering the phenomena of failure localization in the strain softening stage. And most of the mesoscopic models are too complex to be used in the analysis of practical problems directly. The macroscopic constitutive relationship which is capable of describing the mesoscopic damage mechanism and considering the convenience of application needs to be established ${ }^{[10]}$.

The constitutive model based on strain strength distribution ${ }^{[11-13]}$ was proposed in order to establish the relationship between mesoscopic damage and macroscopic property. Strain is the strength measuring index and the material properties are described by the distribution density function. Fracture state is expressed by the fracture factor. Considering the internal heterogeneity of strain strength and the friction on fractured micro planes, the macroscopic mechanical behavior of nonlinearity and strain softening can be obtained naturally.

The objective of this paper is to introduce a statistic damage model to describe the constitutive relationship for the tensile failure process of rock and concrete materials. It is a three-stage model for the representative volume element (RVE) based on strain strength distribution. Considering the mesoscopic damage and fracture mechanism in the process of tensile failure, the relationship between mesoscopic damage and macroscopic property is established by using the strain strength distribution model and experimental stress-strain curves. In this model, the complete stress-strain curve is divided into three parts, including the linear part, the nonlinear part and the strain softening part. A modified plan of softening strain for the stress-strain curve is introduced in this paper based on the traditional modified plan $^{[14,15]}$ so that the strain softening property can be described in a unified form. The parameters of distribution density function of tensile strain strength can be obtained by means of curve fitting and then the whole failure process can be described quantitatively.

\section{Statement of the Problem}

The occurrence and development of damage and fracture in the test specimen can be observed in both laboratory experiments and numerical simulations. New cracks do not initiate in the early stage of the loading process. Damage occurs and grows when the load exceeds the linear elastic limit. And then the micro cracks grow randomly all over the specimen. The principal crack forms in the localization band as the load continues to increase. In this process, the damage mode changes from the uniform body damage to the localization damage. The peak stress is the feature point of the change and the indication of the occurrence of failure. Generally, the failure process mentioned above can be divided into three stages, as shown in Fig.1.

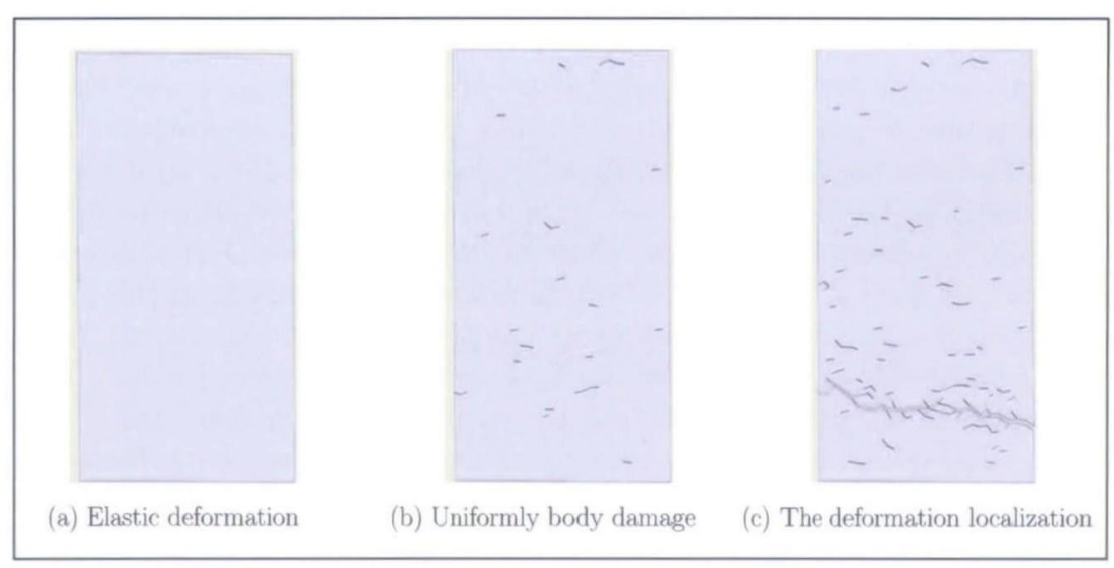

Fig. 1. The fracture state during failure process. (a) No damage takes place in the elastic deformation stage, (b) Damage occurs and micro cracks grow randomly all over the specimen in the uniform damage stage, (c) A principal crack forms in a finite narrow region because of the damage localization. 
The complete uniaxial tensile stress-strain curve of rock and concrete materials usually contains three parts, i.e. the linear part, nonlinear part and strain softening part. The typical complete uniaxial tensile stress-strain curve is shown in Fig.2. Those three parts characterize the three different stages in the deformation and failure process.

In the linear stage, the deformation of the material is linear elastic and the stress-strain curve is linear, shown as part $A B$ in Fig.2. No damage occurs in this stage.

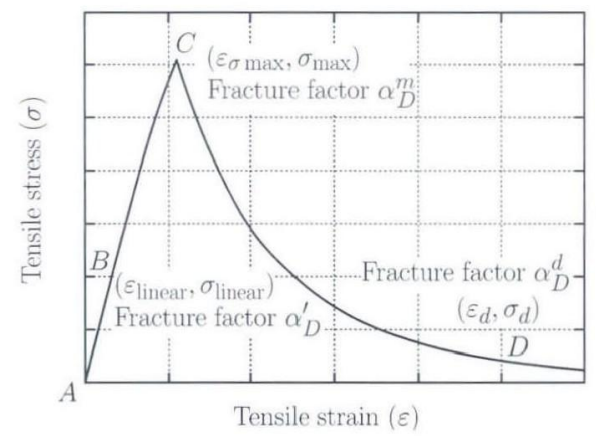

Fig. 2. The typical stress-strain curve of uniaxial tensile test. It contains three parts, the linear part $A B$, nonlinear part $B C$ and strain softening part $C D$. The linear proportional limit is point $B$ and the stress and strain are $\sigma_{\text {linear }}$ and $\varepsilon_{\text {linear }}$. Point $C$ is the peak stress. At this point, the stress and strain are $\sigma_{\max }$ and $\varepsilon_{\sigma \max }$. Point $D$ is the end point of the stress-strain curve. The stress and strain at this point are $\sigma_{d}$ and $\varepsilon_{d}$. The fracture factor values in the states of $B, C$, and $D$ are $\alpha_{D}^{l}, \alpha_{D}^{m}$ and $\alpha_{D}^{d}$.

When the deformation of the material exceeds the linear proportional limit, the nonlinear stage begins. The linear proportional limit is point $B$ and the corresponding stress and strain are $\sigma_{\text {linear }}$ and $\varepsilon_{\text {linear }}$, respectively. The fracture factor is used to express the degree of damage. At point $B$, the linear proportional limit, the fracture factor is $\alpha_{D}^{l}$, which is equal to zero. In the nonlinear part, the damage grows in the body of the specimen, leading to the degradation of macroscopic properties. The stress-strain curve exhibits nonlinear characteristics.

Point $C$ is the peak stress. At this point, the stress and strain are $\sigma_{\max }$ and $\varepsilon_{\sigma \max }$, respectively, and the fracture factor is $\alpha_{D}^{m}$. After the peak stress is reached, the deformation and damage grow in the localization band. Finally, a principal crack forms and the stress-strain relationship exhibits strainsoftening. Point $D$ is the end point of the stress-strain curve. The stress and strain at this point are $\sigma_{d}$ and $\varepsilon_{d}$, respectively.

\section{Tensile Failure Based on the Original Model of Strain Strength Distribution}

The strain strength distribution model is used to describe the heterogeneous characteristics of rock and concrete materials and to establish the relationship between the macroscopic properties and the mesoscopic damage. The assumption of strain strength distribution is the major feature of this model. The strain strength complies with a certain distribution law in the representative volume element. Any section or plane in the representative volume element can be composed of elastic micro planes and fractured micro planes, as shown in Fig.3. The interactions remain elastic on the elastic micro planes, but turn into contact on the fractured micro planes. The tensile tress on fractured micro planes is zero. The interactions on fractured micro planes comply with the Coulomb's friction law. The expressions for the mechanical behaviors of microplanes on the fracture plane have been discussed by $\mathrm{Li}$ and $\mathrm{Zhou}{ }^{[2,13]}$.

Considering the tensile failure behavior in the original model of strain strength distribution, a tensile plane may be composed of the intact part and the fractured part in the progressive process of tensile failure. The intact part consists of elastic micro planes whose tensile strains are always below their tensile strain strengths. The fractured part consists of fractured micro planes whose tensile strains exceed their tensile strain strengths. The elastic micro planes remain linear elastic, but the fractured micro planes can bear no more tension. The fracture factor $\alpha_{D}$ and the intact factor $\alpha_{I}$ are defined in 


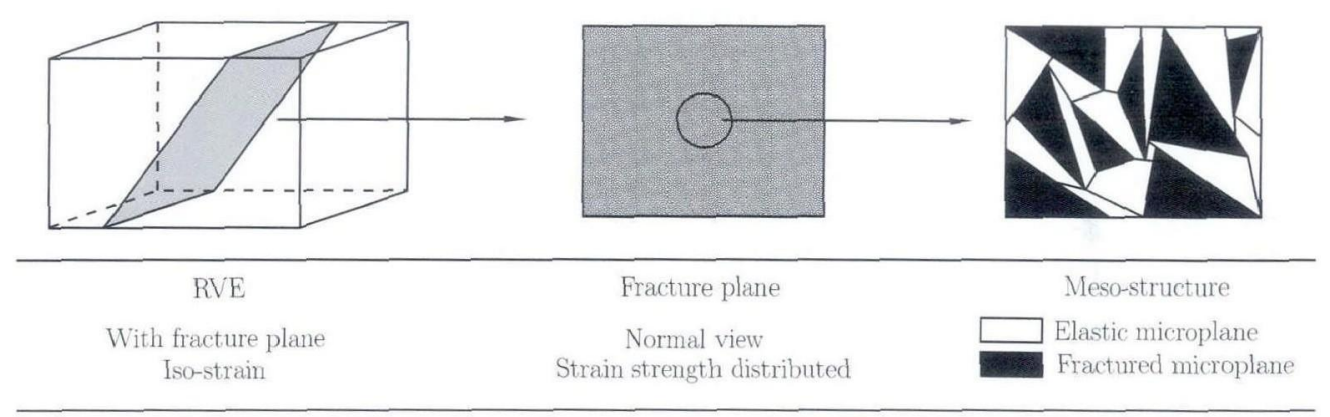

Fig. 3. Elastic microplanes and fractured microplanes on the fracture plane in RVE.

the model to represent the damage state of the tensile fracture plane. $\alpha_{D}$ is expressed as

$$
\alpha_{D}=\frac{S_{b}}{S_{p}}=\frac{\int_{\varepsilon_{\min }}^{\bar{\varepsilon}} f(\varepsilon) \mathrm{d} \varepsilon}{\int_{\varepsilon_{\min }}^{\varepsilon_{\max }} f(\varepsilon) \mathrm{d} \varepsilon}
$$

where $S_{b}$ is the damage area, $S_{p}$ is the total area, $\bar{\varepsilon}$ is the maximum tensile strain in history, $\varepsilon_{\min }$ is the linear limit strain, $\varepsilon_{\max }$ is the failure strain strength, $\varepsilon$ is the integral variable, and $f(\varepsilon)$ is the distribution density function of tensile strain.

The fracture factor $\alpha_{D}$ and the intact factor $\alpha_{I}$ are used to describe the damage state, and the values of $\alpha_{I}$ and $\alpha_{D}$ are both between 0 and 1 , which satisfy

$$
\alpha_{I}+\alpha_{D}=1
$$

If $\bar{\varepsilon}$ is less than the value of the tensile strain strength $\varepsilon_{\min }, \alpha_{D}$ is equal to 0 and $\alpha_{I}$ is equal to 1 , which means no damage occurs. If $\bar{\varepsilon}$ is larger than the value of the tensile strain strength $\varepsilon_{\max }, \alpha_{D}$ is equal to 1 and $\alpha_{I}$ is equal to 0 , which means the tensile plane is totally fractured. Otherwise, the tensile plane is partly fractured, and $\alpha_{D}$ can be expressed as the integration of distribution density function of the tensile strain strength from $\varepsilon_{\min }$ to $\bar{\varepsilon}$. The fracture state of the material can be expressed by the fracture factor $\alpha_{D}$.

Different distribution laws of the tensile strain strength can be applied according to different material properties. The material property can be described by the distribution density function of tensile strain strength $f(\varepsilon)$.

With the intact factor $\alpha_{I}$ and the fracture factor $\alpha_{D}$ defined above, the stress-strain relationship on the tensile plane can be written as

$$
\sigma_{n}=\alpha_{I} E \varepsilon+ \begin{cases}\left(1-\alpha_{I}\right) E \varepsilon & (\varepsilon<0) \\ 0 & (\varepsilon \geq 0)\end{cases}
$$

where $E$ is Young's modulus, $\sigma_{n}$ is the effective normal stress on the tensile plane, and $E \varepsilon$ is an abbreviated expression of the elastic constitutive equation of $E \varepsilon=2 \mu \varepsilon_{n}+\lambda_{0} e$, in which $\mu$ and $\lambda_{0}$ are Lame constants, $\varepsilon_{n}$ is the normal strain, and $e$ is the body strain. The typical complete stress-strain curve of tensile failure is shown in Fig.4.

\section{The Expression of the Three-Stage Model for Failure Process}

A three-stage model is proposed to describe the three stages of the failure process for rock and concrete materials. The linear part, nonlinear part and strain softening part of the stress strain curve are expressed separately. The difference between the three-stage model and the original model is shown in Fig.5. 


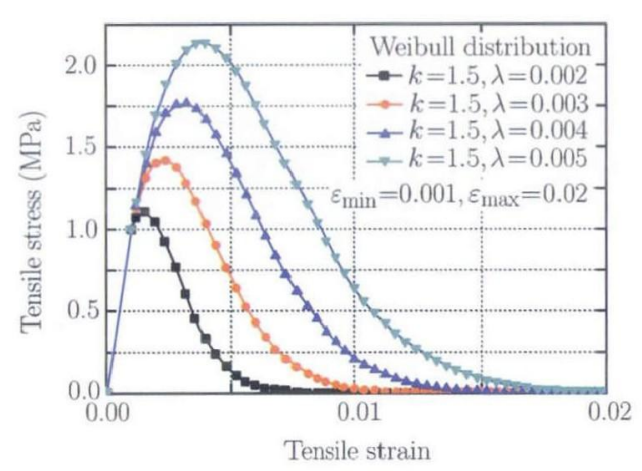

Fig. 4. The stress-strain curves of uniaxial tensile by the original model of strain strength distribution $[12,13]$. $\varepsilon_{\min }$ is the linear limit. When the strain exceeds this point, the stress-strain curve will be nonlinear. $\varepsilon_{\max }$ is the tensile strain strength. When the strain exceeds this point, complete failure of the material takes place. $\lambda$ and $k$ are the shape parameter and scale parameter of Weibull distribution, respectively. $k$ determines the shape of the curve and $\lambda$ determines the peak value and the scale of the curve ${ }^{[12,13]}$.

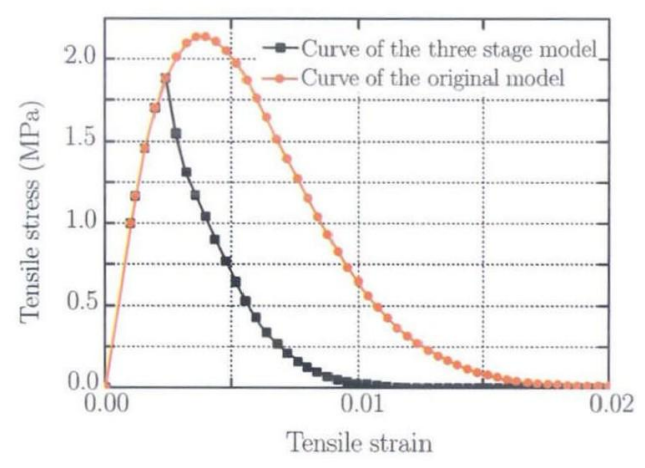

Fig. 5. Comparison of the stress-strain curves between the three-stage model and the original model. Those two curves are overlapped before the peak stress is reached. The nonlinear part of the three-stage model is part of the original model and $\varepsilon_{\min }=\varepsilon_{\text {linear }}, \varepsilon_{\max }$ is the failure strain in the original model of strain strength distribution. It cannot be obtained from the experimental stress-strain curve.

\subsection{Expressions of the fracture factor and the intact factor with Weibull distribution}

Supposing that the strain strength complies with the Weibull distribution law, the distribution density function is expressed as

$$
f(\varepsilon)=\frac{k}{\lambda}\left(\frac{\varepsilon}{\lambda}\right)^{k-1} e^{-(\varepsilon / \lambda)^{k}}
$$

where $f(\varepsilon)$ is the distribution density function of tensile strain strength, $\varepsilon$ is strain, $\lambda$ and $k$ are the shape parameter and scale parameter of Weibull distribution law, respectively.

According to formula (1), the fracture factor $\alpha_{D}$ can be written as

$$
\alpha_{D}=\frac{e^{-(\varepsilon / \lambda)^{k}-e^{-\left(\varepsilon_{\min } / \lambda\right)^{k}}}}{e^{-\left(\varepsilon_{\max } / \lambda\right)^{k}}-e^{-\left(\varepsilon_{\min } / \lambda\right)^{k}}} \quad\left(\varepsilon_{\min } \leq \varepsilon \leq \varepsilon_{\max }\right)
$$

and the intact factor $\alpha_{I}$ can be written as

$$
\alpha_{I}=1-\alpha_{D}=\frac{e^{-\left(\varepsilon_{\max } / \lambda\right)^{k}}-e^{-(\varepsilon / \lambda)^{k}}}{e^{-\left(\varepsilon_{\max } / \lambda\right)^{k}}-e^{-\left(\varepsilon_{\min } / \lambda\right)^{k}}} \quad\left(\varepsilon_{\min } \leq \varepsilon \leq \varepsilon_{\max }\right)
$$

Considering the mesoscopic damage and fracture mechanism in the process of tensile failure, the complete stress-strain curve should be divided into three parts, the linear part $A B$, the nonlinear part $B C$, and the strain softening part $C D$, as shown in Fig.2. The nonlinear part $B C$ represents the mesoscopic damage. The mechanism is body damage. The strain softening part $C D$ represents damage of the localization band. Supposing that the strain strength in each part complies with the Weibull distribution law, then each part of the model is introduced respectively as follows.

\subsection{Linear part}

Deformation of the material is linear elastic in the linear part $A B$. No damage would generate during this stage.

In the linear part, the fracture factor is zero, and the relationship between strain and stress can be written as

$$
\sigma=E \varepsilon
$$

\subsection{Nonlinear part}

The stress-strain curve exhibits nonlinearity when the stain exceeds the linear proportional limit strain $\varepsilon_{\text {linear. }}$ Damage occurs and microcracks develope all over the specimen in the nonlinear part $B C$. The homogeneous damage within the material body takes place. The macroscopic effective modulus 
reduces because of the damage and microcracks. The fracture state in this stage can be characterized by the fracture factor $\alpha_{D}$ which is controlled by the distribution density function of tensile strain strength $f(\varepsilon)$.

In the nonlinear part, the body fracture factor can be written as

$$
\alpha_{D}=\frac{e^{-\left(\varepsilon / \lambda_{1}\right)^{k_{1}}}-e^{-\left(\varepsilon_{\min } / \lambda_{1}\right)^{k_{1}}}}{e^{-\left(\varepsilon_{\max } / \lambda_{1}\right)^{k_{1}}}-e^{-\left(\varepsilon_{\min } / \lambda_{1}\right)^{k_{1}}}} \quad\left(\varepsilon_{\text {linear }} \leq \varepsilon \leq \varepsilon_{\sigma \max }\right)
$$

where $\varepsilon_{\min }$ is the linear proportional limit strain which can be obtained from the stress-strain curve $\varepsilon_{\min }=\varepsilon_{\text {linear. }} . \varepsilon_{\max }$ is the failure strain strength in the original model. But it is unknown in the threestage model because of the change of failure mode. $\lambda_{1}$ and $k_{1}$ are the parameters of Weibull distribution in the nonlinear part which can be obtained by means of curve fitting. $\varepsilon_{\sigma \max }$ is the strain value at the peak stress.

In order to obtain the fracture factor $\alpha_{D}$ in the nonlinear part, both the numerator and the denominator of formula (8) are multiplied by $\left(e^{-\left(\varepsilon_{\sigma \max } / \lambda_{1}\right)^{k_{1}}}-e^{-\left(\varepsilon_{\text {linear }} / \lambda_{1}\right)^{k_{2}}}\right)$, and $\alpha_{D}$ can then be expressed as

$$
\alpha_{D}=\frac{e^{-\left(\varepsilon_{\sigma \max } / \lambda_{1}\right)^{k_{1}}}-e^{-\left(\varepsilon_{\text {linear }} / \lambda_{1}\right)^{k_{1}}}}{e^{-\left(\varepsilon_{\max } / \lambda_{1}\right)^{k_{1}}}-e^{-\left(\varepsilon_{\text {linear }} / \lambda_{1}\right)^{k_{1}}}} \times \frac{e^{-\left(\varepsilon / \lambda_{1}\right)^{k_{1}}}-e^{-\left(\varepsilon_{\text {linear }} / \lambda_{1}\right)^{k_{1}}}}{e^{-\left(\varepsilon_{\sigma \max } / \lambda_{1}\right)^{k_{1}}}-e^{-\left(\varepsilon_{\text {linear }} / \lambda_{1}\right)^{k_{1}}}}
$$

The fracture factor at the peak stress $\alpha_{D}^{m}$ can be expressed as

$$
\alpha_{D}^{m}=\frac{e^{-\left(\varepsilon_{\sigma \max } / \lambda_{1}\right)^{k_{1}}}-e^{-\left(\varepsilon_{\text {linear }} / \lambda_{1}\right)^{k_{1}}}}{e^{-\left(\varepsilon_{\max } / \lambda_{1}\right)^{k_{1}}}-e^{-\left(\varepsilon_{\text {linear }} / \lambda_{1}\right)^{k_{1}}}}
$$

Then expression (9) can be written as

$$
\alpha_{D}=\alpha_{D}^{m} \frac{e^{-\left(\varepsilon / \lambda_{1}\right)^{k_{1}}}-e^{-\left(\varepsilon_{\text {linear }} / \lambda_{1}\right)^{k_{1}}}}{e^{-\left(\varepsilon_{\sigma \max } / \lambda_{1}\right)^{k_{1}}}-e^{-\left(\varepsilon_{\text {linear }} / \lambda_{1}\right)^{k_{1}}}} \quad\left(\varepsilon_{\text {linear }} \leq \varepsilon \leq \varepsilon_{\sigma \max }\right)
$$

According to formula (3) and the value of fracture factor, $\alpha_{D}^{m}$ at the peak stress can be expressed as

$$
\sigma_{\max }=\left(1-\alpha_{D}^{m}\right) E \varepsilon_{\sigma \max }
$$

$\alpha_{D}^{m}$ is obtained from formula (12) and expressed as

$$
\alpha_{D}^{m}=1-\frac{\sigma_{\max }}{E \varepsilon_{\sigma \max }}
$$

\subsection{Strain softening part}

The stress-strain curve exhibits strain softening when the strain exceeds $\varepsilon_{\sigma \text { max }}$ at the peak stress. A localization band forms in this stage, and damage grows primarily in the localization band. The peak stress is the start of this stage. The fracture factor $\alpha_{D}$ represents the damage degree of the localization band, which can be used to represent the fracture state in the strain softening stage. In this stage, the strain used in the expression should not be the global strain of the RVE. Strain of the localization band must be used to express the localization behavior.

In the strain softening part, the fracture factor $\alpha_{D}$ in the localization band can be written as

$$
\alpha_{D}=\frac{e^{-\left(\varepsilon / \lambda_{2}\right)^{k_{2}}}-e^{-\left(\varepsilon_{\min } / \lambda_{2}\right)^{k_{2}}}}{e^{-\left(\varepsilon_{\max } / \lambda_{2}\right)^{k_{2}}}-e^{-\left(\varepsilon_{\min } / \lambda_{2}\right)^{k_{2}}}} \quad\left(\varepsilon_{\sigma \max } \leq \varepsilon \leq \varepsilon_{d}\right)
$$

And it can also be expressed as

$$
\alpha_{D}=1-\frac{e^{-\left(\varepsilon_{\max } / \lambda_{2}\right)^{k_{2}}}-e^{-\left(\varepsilon / \lambda_{2}\right)^{k_{2}}}}{e^{-\left(\varepsilon_{\max } / \lambda_{2}\right)^{k_{2}}}-e^{-\left(\varepsilon_{\min } / \lambda_{2}\right)^{k_{2}}}} \quad\left(\varepsilon_{\sigma \max } \leq \varepsilon \leq \varepsilon_{d}\right)
$$

where $\varepsilon_{\min }$ and $\varepsilon_{\max }$ are the minimum strain strength and the maximum strain strength of the localization band, respectively. $\lambda_{2}$ and $k_{2}$ are the parameters of Weibull distribution in the strain softening part, $\varepsilon_{\sigma \max }$ is the strain of the peak stress. 
In order to obtain the expression of fracture factor $\alpha_{D}$ in the localization band, both the numerator and the denominator of the second part in formula (14) are multiplied by $\left(e^{-\left(\varepsilon_{\max } / \lambda\right)^{k}}-e^{-\left(\varepsilon_{\sigma \max } / \lambda\right)^{k}}\right)$, and the fracture factor $\alpha_{D}$ is then expressed as

$$
\alpha_{D}=1-\frac{e^{-\left(\varepsilon_{\max } / \lambda_{2}\right)^{k_{2}}}-e^{-\left(\varepsilon_{\sigma \max } / \lambda_{2}\right)^{k_{2}}}}{e^{-\left(\varepsilon_{\max } / \lambda_{2}\right)^{k_{2}}}-e^{-\left(\varepsilon_{\min } / \lambda_{2}\right)^{k_{2}}}} \times \frac{e^{-\left(\varepsilon_{\max } / \lambda_{2}\right)^{k_{2}}}-e^{-\left(\varepsilon / \lambda_{2}\right)^{k_{2}}}}{e^{-\left(\varepsilon_{\max } / \lambda_{2}\right)^{k_{2}}}-e^{-\left(\varepsilon_{\sigma \max } / \lambda_{2}\right)^{k_{2}}}}
$$

At the peak stress, the value of fracture factor $\alpha_{D}$ can be expressed as

$$
\alpha_{D}^{m}=1-\frac{e^{-\left(\varepsilon_{\max } / \lambda_{2}\right)^{k_{2}}}-e^{-\left(\varepsilon_{\sigma \max } / \lambda_{2}\right)^{k_{2}}}}{e^{-\left(\varepsilon_{\max } / \lambda_{2}\right)^{k_{2}}}-e^{-\left(\varepsilon_{\min } / \lambda_{2}\right)^{k_{2}}}}
$$

At point $D$ the value of fracture factor $\alpha_{D}$ can be expressed as

$$
\alpha_{D}^{d}=1-\frac{e^{-\left(\varepsilon_{\max } / \lambda_{2}\right)^{k_{2}}}-e^{-\left(\varepsilon_{d} / \lambda_{2}\right)^{k_{2}}}}{e^{-\left(\varepsilon_{\max } / \lambda_{2}\right)^{k_{2}}}-e^{-\left(\varepsilon_{\min } / \lambda_{2}\right)^{k_{2}}}}
$$

According to expressions (16), (17) and (18), the localization band fracture factor $\alpha_{D}$ can be expressed as

$$
\alpha_{D}=1-\frac{\left(\alpha_{D}^{d}-\alpha_{D}^{m}\right) e^{-\left(\varepsilon / \lambda_{2}\right)^{k_{2}}}+\left(1-\alpha_{D}^{d}\right) e^{-\left(\varepsilon_{\sigma \max } / \lambda_{2}\right)^{k_{2}}}-\left(1-\alpha_{D}^{m}\right) e^{-\left(\varepsilon_{d} / \lambda_{2}\right)^{k_{2}}}}{e^{-\left(\varepsilon_{\sigma \max } / \lambda_{2}\right)^{k_{2}}}-e^{-\left(\varepsilon_{d} / \lambda_{2}\right)^{k_{2}}}} \quad\left(\varepsilon_{\sigma \max }<\varepsilon \leq \varepsilon_{d}\right)
$$

The fracture factor $\alpha_{D}$ in localization band can be obtained if $\alpha_{D}^{d}$ is known. At point $D$, expression (3) can be written as

$\alpha_{D}^{d}$ can be expressed as

$$
\sigma_{d}=\left(1-\alpha_{D}^{d}\right) E \varepsilon_{d}
$$

$$
\alpha_{D}^{d}=1-\frac{\sigma_{d}}{E \varepsilon_{d}}
$$

\subsection{Three-stage model for tensile failure process}

Considering the tensile failure behavior in the three-stage model of strain strength distribution, the stress-strain relationship can be

$$
\sigma=\left(1-\alpha_{D}\right) E \varepsilon
$$

where the fracture factor $\alpha_{D}$ can be expressed as

$$
\alpha_{D}=\left\{\begin{array}{lc}
0 & \left(\varepsilon \leq \varepsilon_{\text {linear }}\right) \\
\alpha_{D}^{m} \frac{e^{-\left(\varepsilon / \lambda_{1}\right)^{k_{1}}}-e^{-\left(\varepsilon_{\text {linear }} / \lambda_{1}\right)^{k_{1}}}}{e^{-\left(\varepsilon_{\sigma \max } / \lambda_{1}\right)^{k_{1}}-e^{-\left(\varepsilon_{\text {linear }} / \lambda_{1}\right)^{k_{1}}}}} & \left(\varepsilon_{\text {linear }} \leq \varepsilon \leq \varepsilon_{\sigma \max }\right) \\
1-\frac{\left(\alpha_{D}^{d}-\alpha_{D}^{m}\right) e^{-\left(\varepsilon / \lambda_{2}\right)^{k_{2}}}+\left(1-\alpha_{D}^{d}\right) e^{-\left(\varepsilon_{\sigma \max } / \lambda_{2}\right)^{k_{2}}}-\left(1-\alpha_{D}^{m}\right) e^{-\left(\varepsilon_{d} / \lambda_{2}\right)^{k_{2}}}}{e^{-\left(\varepsilon_{\sigma \max } / \lambda_{2}\right)^{k_{2}}}-e^{-\left(\varepsilon_{d} / \lambda_{2}\right)^{k_{2}}}} & \left(\varepsilon_{\sigma \max }<\varepsilon \leq \varepsilon_{d}\right)
\end{array}\right.
$$

So the fracture factor function $\alpha_{D}$ is a piecewise function with different parts representing different modes of damage. The first part represents the state of linear elastic deformation and there is no damage in the specimen. The second part describes the uniform damage of the material body and represents the damage degree of the representative volume element. The third part describes the evolution of the localization damage and represents the damage degree of the localization band in the representative volume element. The linear proportional limit and the peak stress are key points to determine the fracture factor $\alpha_{D}$. The damage occurs and micro cracks begin to initiate when the strain exceeds the linear limit strain. So the point of linear proportional limit can be considered as the end of the linear elastic deformation and the beginning of the uniform body damage. The peak stress is the turning point from the uniform damage mode of the material body to the localization damage mode.

The inhomogeneity of rock and concrete materials is considered in the three-stage model and is expressed by the distribution density function of strain strength. The distribution density function of strain strength controls the development of damage in the specimen and the change of damage mode from the homogeneous body damage to localization. The distribution density function of strain strength 
$f(\varepsilon)$ can be expressed as the derivation of the fracture factor on the strain according to equation (23), and written as

$$
f(\varepsilon)= \begin{cases}0 & \left(\varepsilon \leq \varepsilon_{\text {linear }}\right) \\ \frac{\alpha_{D}^{m}}{e^{-\left(\varepsilon_{\text {linear }} / \lambda_{1}\right)^{k_{1}}}-e^{-\left(\varepsilon_{\sigma \max } / \lambda_{1}\right)^{k_{1}}}}\left(\frac{k_{1}}{\lambda_{1}}\right)\left(\frac{\varepsilon}{\lambda_{1}}\right)^{k_{1}-1} e^{-\left(\varepsilon / \lambda_{1}\right)^{k_{1}}} & \left(\varepsilon_{\text {linear }} \leq \varepsilon \leq \varepsilon_{\sigma \max }\right) \\ \frac{\alpha_{D}^{d}-\alpha_{D}^{m}}{e^{-\left(\varepsilon_{d} / \lambda_{2}\right)^{k_{2}}}-e^{-\left(\varepsilon_{\sigma \max } / \lambda_{2}\right)^{k_{2}}}}\left(\frac{k_{2}}{\lambda_{2}}\right)\left(\frac{\varepsilon}{\lambda_{2}}\right)^{k_{2}-1} e^{-\left(\varepsilon / \lambda_{2}\right)^{k_{2}}} & \left(\varepsilon_{\sigma_{\max }} \leq \varepsilon \leq \varepsilon_{d}\right)\end{cases}
$$

where $\lambda_{1}$ and $k_{1}$ are the parameters of the distribution density function of tensile strain strength in the material body in the nonlinear stage; and $\lambda_{2}$ and $k_{2}$ are the parameters of the distribution density function of tensile strain strength in the localization band in the strain softening stage.

\subsection{Determination of the parameters}

Some of the parameters in the three-stage model can be directly obtained from the experimental complete stress-strain curves, which include the linear proportional limit strain $\varepsilon_{\text {linear }}$ in the nonlinear part, the strain at the peak stress $\varepsilon_{\sigma \max }$, and the end point of the experimental curve $\varepsilon_{d}$. The fracture factor value at the peak stress $\alpha_{D}^{m}$ and the fracture factor $\alpha_{D}^{d}$ at point $D$ can be obtained from the data of the stress-strain curve according to formulas (13) and (21).

Other parameters in this model cannot be directly obtained from the experimental stress-strain curves, which include $\lambda_{1}, k_{1}, \lambda_{2}$ and $k_{2}$. However, these parameters can be obtained by means of curve fitting based on the nonlinear least square method ${ }^{[16-18]}$

\section{Applications and Verification}

\subsection{Modification of the softening strain of the complete stress-strain curve}

A closed-loop servo-controlled testing machine ${ }^{[15,19]}$ was used to obtain the complete stress-strain curve in uniaxial tension experiment. Loading rate was controlled by the rate of fracture propagation in the strain softening stage. Damage developed in the localization band while elastic unloading took place in other parts of the specimen simultaneously. The increment of the deformation was provided entirely by the deformation of the localization band.

The macroscopic strain measured by the testing machine was affected by the incremental deformation of the localization band and the deformation recovery of the non-localized parts was disregarded. So the macroscopic strain measured could not reflect the real deformation of localization band ${ }^{[14,15]}$. It is necessary to modify the softening stain, in order to express the deformation of localization band. The basic idea of the modification method is based on the assumption that the damage only takes place on the localization band during the localization damage stage, so that the strain of localization band can be easily determined through the global strain and the strain recovery of the non-localized parts.

The typical stress-strain curve is shown in Fig.6 . Point $Q$ is in the strain softening part. The stress and strain at point $Q$ are $\sigma_{0}$ and $\varepsilon_{0}$, respectively. Point $Q$ changes to point $Q^{\prime}$ after modification. The corresponding stress and strain are $\sigma_{0}$ and $\varepsilon_{0}^{\prime}$, respectively. The macroscopic deformation of specimen $\delta$ at point $Q$ can be expressed as

$$
\delta=\delta_{\sigma \max }-\Delta \varepsilon l+\Delta \delta
$$

where $\delta$ is the deformation measured by the testing machine; $\delta_{\sigma \max }$ is the deformation of the specimen at the peak stress; $\Delta \varepsilon l$ is the unloading deformation of the elastic parts; $l$ is the effective length of the specimen; and $\Delta \delta$ is the incremental deformation of the localization band.

Formula (25) can be further expressed in the form of strain as

$$
\varepsilon_{0}=\varepsilon_{\sigma \max }-\Delta \varepsilon+\delta \varepsilon
$$

where $\varepsilon_{0}$ is the macroscopic strain measured by the testing machine; $\varepsilon_{\sigma \max }$ is the strain at the peak stress; $\Delta \varepsilon$ is the strain of unloading; and $\delta \varepsilon$ is the effective incremental strain of the localization band.

For the strain of localization band, the deformation recovered from the unloading of non-localized parts should not be considered. The modified strain of the localization band can be written as

$$
\varepsilon_{0}^{\prime}=\varepsilon_{\sigma \max }+\delta \varepsilon
$$




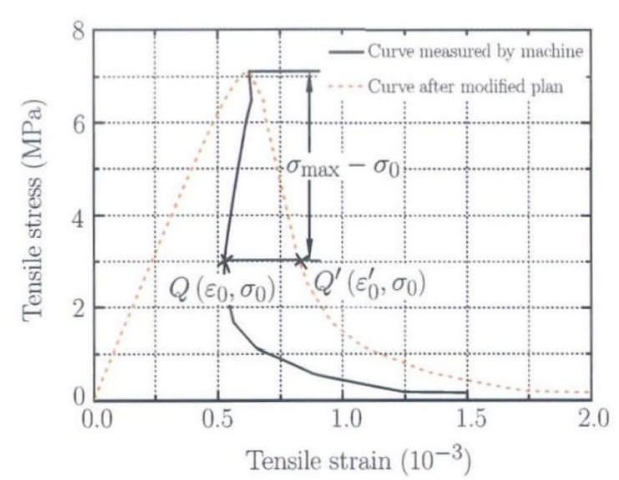

Fig. 6. The typical stress-strain curves before and after modification.

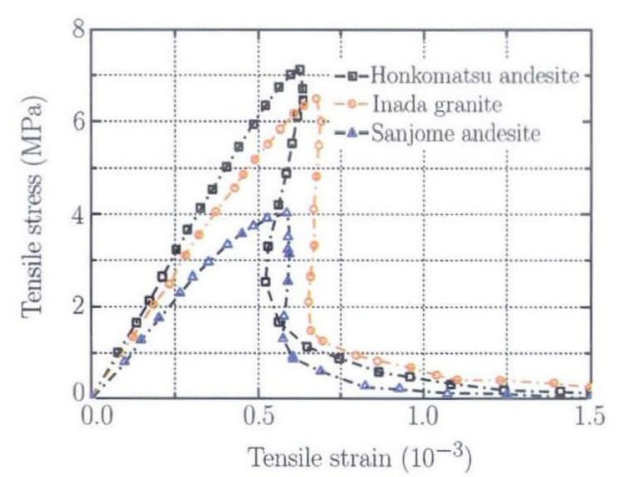

Fig. 7. Experimental complete stress-strain curves of uniaxial tension ${ }^{[19]}$. Three typical rocks, honkomatsu andesite, inada granite and sanjome andesite, were tested by Okubo,S. and Fukui,K. The stress-strain curves of those three typical rocks are very similar in shape and the strain softening parts are very steep.

According to expression (26), $\varepsilon_{0}^{\prime}$ should be further written as

$$
\varepsilon_{0}^{\prime}=\varepsilon_{0}+\Delta \varepsilon
$$

Where $\Delta \varepsilon$ can be written as

$$
\Delta \varepsilon=\frac{\sigma_{\max }-\sigma_{0}}{\left(1-\alpha_{D}^{m}\right) E}
$$

The new point $Q^{\prime}$ can be obtained after modification and then the macroscopic strain can reflect the real deformation of localization band. The incremental strain is entirely provided by the localization band.

The experimental results of uniaxial tensile tests by Okubo,S. and Fukui,K. ${ }^{[19]}$ are shown in Fig.7. The modification is used in those experimental results, and the complete stress-strain curves of uniaxial tensile tests after modification are shown in Fig.8. The strain softening parts after modification become less steep.

\subsection{Determination of the distributive parameters for different rock materials}

The modified experimental complete stress-strain curves of uniaxial tension in Fig. 8 are used as an example. The parameters for the three types of rock are listed in Table 1. Although the peak stress of Honkomatsu andesite is considerably higher than that of Sanjome andesite, there is little difference in the shapes of the stress-strain curves for the two sandstones. Each curve is divided into three parts according to the three-stage model, including the linear part, nonlinear part and strain softening part.

$\varepsilon_{\text {linear }}, \varepsilon_{\sigma \max }$ and $\varepsilon_{d}$ are directly obtained from the stress-strain curves. $\alpha_{D}^{m}$ and $\alpha_{D}^{d}$ are obtained by expressions (13) and (21). $\lambda_{1}, k_{1}, \lambda_{2}$ and $k_{2}$ are obtained by means of curve fitting based on the nonlinear least square method. The parameters obtained are listed in Table 1.

\begin{tabular}{|c|c|c|c|c|c|c|c|c|c|c|}
\hline Specimen number & $E$ & $\varepsilon_{\text {linear }}$ & $\varepsilon_{\sigma \max }$ & $\varepsilon_{d}$ & $\alpha_{D}^{m}$ & $\alpha_{D}^{d}$ & $\lambda_{1}$ & $k_{1}$ & $\lambda_{2}$ & $k_{2}$ \\
\hline Honkomatsu andesite & $1.25 \mathrm{e} 10 \mathrm{~Pa}$ & $4.39 e-4$ & $6.25 e-4$ & $2.01 e-3$ & $8.70 \%$ & $994 \%$ & $6.25 e-2$ & 6.13 & $6.25 \mathrm{e}-6$ & 0.445 \\
\hline Inada granite & $1.06 \mathrm{e} 10 \mathrm{~Pa}$ & $4.91 e-4$ & $6.78 e-4$ & $2.04 \mathrm{e}-3$ & $9.40 \%$ & $988 \%$ & $6.78 e-2$ & 4.47 & $6.78 e-6$ & 0.430 \\
\hline Sanjome andesite & 8.45e9 $\mathrm{Pa}$ & $3.49 e-4$ & $5.87 e-4$ & $1.89 \mathrm{e}-3$ & $186 \%$ & $100 \%$ & $5.87 e-2$ & 2.60 & $5.87 e-6$ & 0.445 \\
\hline
\end{tabular}

Table 1. The tensile property parameters of different rock types

The theoretical complete stress-strain curves of Honkomatsu andesite, Inada granite and Sanjome andesite obtained from the three stage model are shown in Fig.9. The elastic moduli of Honkomatsu andesite, Inada granite and Sanjome andesite are $1.25 \mathrm{e} 10 \mathrm{~Pa}, 1.06 \mathrm{e} 10 \mathrm{~Pa}$ and $8.49 \mathrm{e} 9 \mathrm{~Pa}$, respectively. The stress-strain curves show nonlinear properties when the strains exceed the linear proportional limits. The strains of the peak stresses are $6.25 \mathrm{e}-4,5.87 \mathrm{e}-4$ and $6.78 \mathrm{e}-4$, respectively. Strain softening takes 


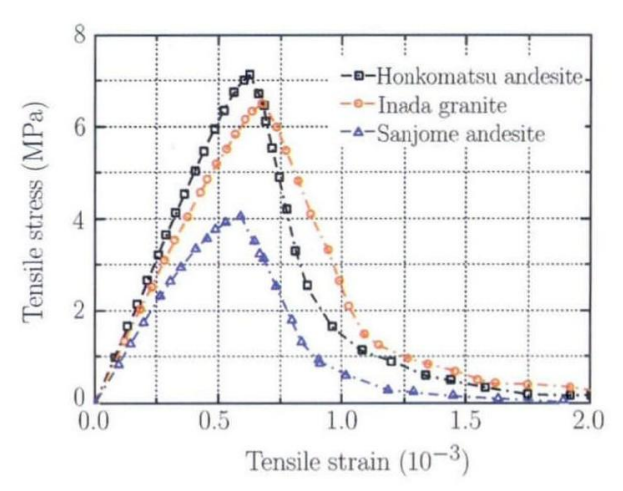

Fig. 8. Complete stress-strain curves of uniaxial tension after modification. The strain softening parts after modification become less steep.

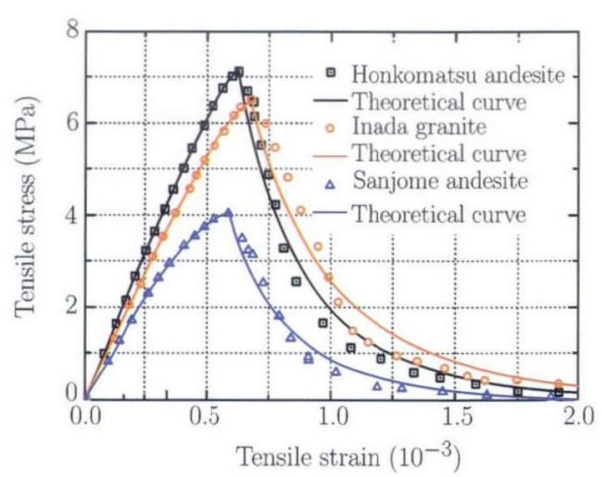

Fig. 9. Comparison of the theoretical results and the experimental complete stress-strain curves. The experimental data is expressed by discrete points and the theoretical curves are expressed by solid lines. The elastic moduli of Honkomatsu andesite, Inada granite and Sanjome andesite are $1.25 \mathrm{e} 10 \mathrm{~Pa}, 1.06 \mathrm{e} 10 \mathrm{~Pa}$ and $8.49 \mathrm{e} 9 \mathrm{~Pa}$, respectively. The stress-strain curve becomes nonlinear when the strain exceeds the linear proportional limit. The strains of the peak stresses are $6.25 \mathrm{e}-4,5.87 \mathrm{e}-4$ and $6.78 \mathrm{e}-4$, respectively. The curve becomes strain softening when the strain exceeds the peak stress.

place when the strain exceeds the peak stress. The theoretical complete stress-strain curves agree with the experimental data very well.

The tensile fracture factors of Honkomatsu andesite, Inada granite and Sanjome andesite are shown in Fig. 10. They are very similar in shape. The fracture factor at the peak stress of Honkomatsu andesite is $8.70 \%$ and that of Inada granite is $9.40 \% . \alpha_{D}^{m}$ of Sanjome andesite is $18.6 \%$, which is larger than the other two. The fracture factor at the peak stress $\alpha_{D}^{m}$ is relatively small, which means that the damage and fracture in the specimen at nonlinear stage are not obvious. But the fracture factor exhibits a rapid increase when the strain exceeds the peak stress.

The density functions of tensile strain strength distribution for Honkomatsu andesite, Inada granite and Sanjome andesite are shown in Fig.11. They are also very similar in shape. The distribution density is in a low level in the nonlinear part, but in a high level near the peak stress. The density function of tensile strain strength distribution is a monotone increasing function before the peak stress is reached, but decreases monotonously after that. The distribution density function of strain strength is a piecewise function and there is an obvious jump at the peak stress. The obvious jump at the peak stress point is caused by the change of damage mode. The change is from body damage to the localization band damage.

The distribution density function of tensile strain strength can be used to describe the material properties and to determine the failure process of material. The distribution density function of tensile strain strength can be obtained through the derivation of the fracture factor function.

\subsection{Determination of the distributive parameters of concrete material}

The experiment results of uniaxial tensile tests of Wu Feng ${ }^{[15]}$ are shown in Fig.12.

More than one specimen with the same material were tested in order to avoid accidental error in the uniaxial tension tests. In Wu's tests, all the parameters can be obtained by averaging the test results. $\varepsilon_{\text {linear }}, \varepsilon_{\sigma \max }$ and $\varepsilon_{d}$ are directly obtained from the stress-strain curves. $\alpha_{D}^{m}$ and $\alpha_{D}^{d}$ are obtained by expressions (13) and (21). $\lambda_{1}, k_{1}, \lambda_{2}$ and $k_{2}$ are obtained by means of curve fitting based on the nonlinear least square method. The parameters obtained are listed in Table 2.

There is little difference between the parameters of different samples listed in Table 2 . So the parameters of concrete can be determined by averaging the results.

The theoretical complete stress-strain curve and the experimental data of this concrete material are shown in Fig.13. The average elastic modulus in the linear part is $3.20 \mathrm{e} 10 \mathrm{~Pa}$. The average linear 


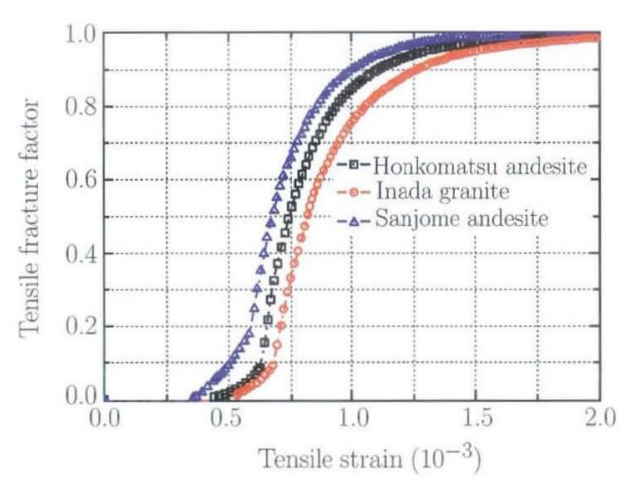

Fig. 10. Curves of the tensile fracture factor for Honkomatsu andesite, Inada granite and Sanjome andesite. The fracture factor at the peak stress of Honkomatsu andesite is $8.70 \%$ and that of Inada granite is $9.40 \%$. $\alpha_{D}^{m}$ of Sanjome andesite is is $18.6 \%$, which is larger than the other two. The damage and fracture grow rapidly near the peak stress.

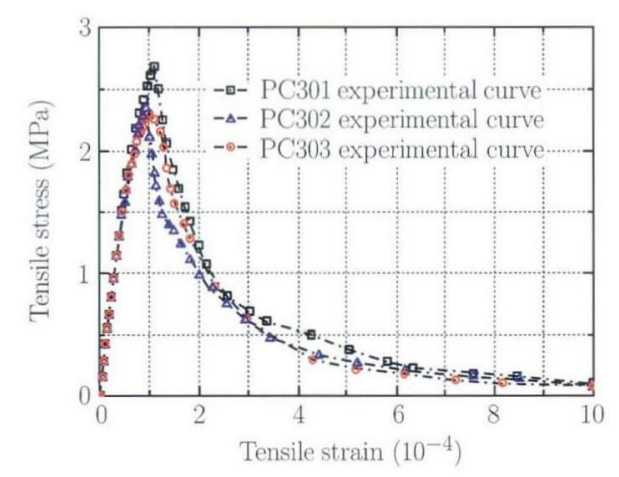

Fig. 12. Complete stress-strain curves of uniaxial tensile tests of $\mathrm{Wu}^{[15]}$.

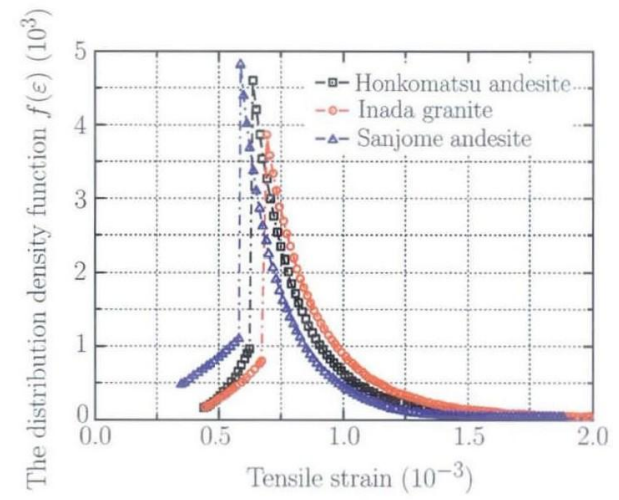

Fig. 11. The distribution density functions of tensile strain strength for Honkomatsu andesite, Inada granite and Sanjome andesite. The distribution density is in a low level in the nonlinear part but in a high level near the peak stress. The distribution density function is a monotone increasing function before the peak stress is reached, but decreases monotonously after that. The tensile strain strength distribution density function is a piecewise function and there is an obvious jump at the peak stress.

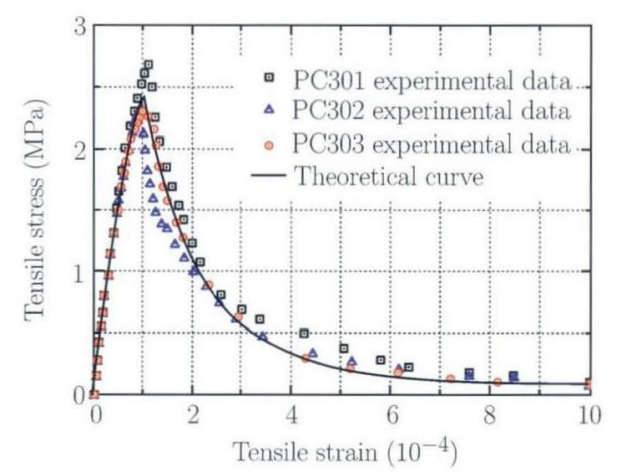

Fig. 13. Complete stress-strain curve of concrete material.

Table 2. The tensile property parameters of different specimens of the same concrete

\begin{tabular}{|c|c|c|c|c|c|c|c|c|c|c|}
\hline Specimen number & $E$ & $\varepsilon_{\text {linear }}$ & $\varepsilon_{\sigma \max }$ & $\varepsilon_{d}$ & $\alpha_{D}^{m}$ & $\alpha_{D}^{d}$ & $\lambda_{1}$ & $k_{1}$ & $\lambda_{2}$ & $k_{2}$ \\
\hline PC301 & $3.2 \mathrm{e} 10 \mathrm{~Pa}$ & $4.1 e-5$ & $1.12 \mathrm{e}-4$ & $1.00 \mathrm{e}-\overline{3}$ & 0.253 & 0.997 & $1.12 \mathrm{e}-2$ & 1.79 & $1.12 \mathrm{e}-6$ & 0.373 \\
\hline PC303 & $3.2 \mathrm{e} 10 \mathrm{~Pa}$ & $4.1 \mathrm{e}-5$ & $1.01 e-4$ & $1.00 \mathbf{e}-3$ & 0.288 & 0.998 & $1.01 \mathrm{e}-2$ & 1.35 & $1.01 \mathrm{e}-6$ & 0.351 \\
\hline
\end{tabular}

proportional limit strain is $4.10 \mathrm{e}-5$. The average strain at peak stress is $1.03 \mathrm{e}-4$. The theoretical complete stress-strain curve agrees with the experimental data very well in each stage.

The tensile fracture factor of this concrete material is shown in Fig.14. The fracture factor at the peak stress is $26.5 \%$. But it increases rapidly after the peak stress is reached.

The distribution density function of tensile strength for the concrete material is shown in Fig.15. The shape of the curve is similar to the distribution density function of rocks.

\section{Conclusions}

The three-stage model for tensile failure process of rock and concrete materials based on strain strength distribution is introduced in this paper. Considering the fracture mode in the tensile failure process, the relationship between mesoscopic damage and macroscopic property are established with the three-stage model. The distributive properties and parameters of rock and concrete materials can 


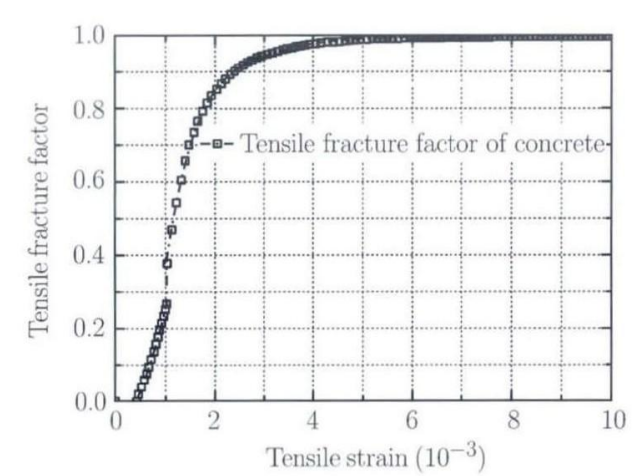

Fig. 14. Tensile Fracture Factor of concrete material. The fracture factor at peak stress is $26.5 \%$. The damage and fracture grow rapidly near the peak stress.

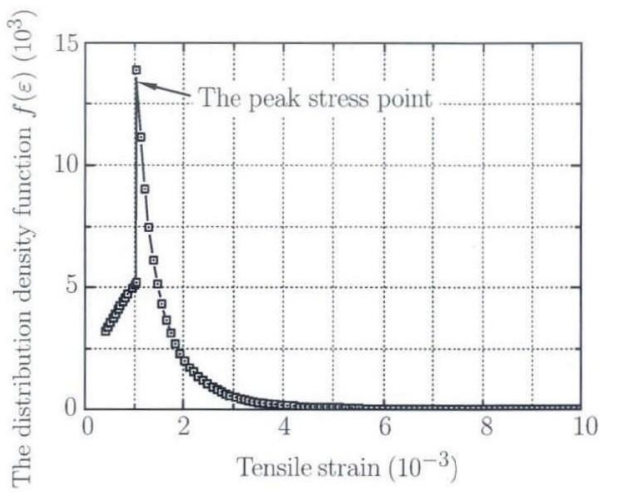

Fig. 15. The distribution density functions of the strain strength for the concrete material.

be obtained through the experimental stress-strain curves. According to the model, the complete stressstrain curve is divided into three parts, i.e. the linear part, nonlinear part and strain softening part, which represent the linear elastic stage, body damage stage and localization band damage stage, respectively. There is no damage generated in the specimen during the first stage. The damage occurs and microcracks grow randomly all over the specimen in the second stage. The peak stress is the beginning of the third stage and a localization band is formed. In the localization stage, the global strain of RVE should not be used to describe the localization damage. Alternatively, the strain of the localization band must be used to express the behavior of strain softening because the failure mode changes from body damage to localization damage. The fracture state in different failure stages and damage modes is described by the fracture factor. The fracture factor is the function of the global strain of RVE in the body damage stage, and the strain of localization band in the localization damage stage.

The method to determine distributive parameters of the model according to the experimental stressstrain curve is introduced. Examples of some practical applications of this model show that distributive parameters and distribution density function in each stage of the model can be effectively obtained with experimental curve. And the theoretical results of the model agree with the test data very well, which has verified the practicability and validity of the model.

In the three-stage model, the fracture state in each stage of the failure process is expressed by the fracture factor. In the applications, the fracture factors of Honkomatsu andesite, Inada granite, Sanjome andesite and the concrete material mentioned above grow rapidly near the peak stress. The strain strength distribution density is in a low level in the nonlinear part but in a high level near the peak stress. It is a piecewise function and there is an obvious jump at the peak stress. The jump at the peak stress point is a signal for the change in damage mode, from body damage to localization band damage.

The internal fracture evolution process and different damage modes in the failure process of material are described with the three stage model. The heterogeneity of rock and concrete materials is described with distributive parameters, which can be obtained through experimental data. Strength distribution is seen as a basic property of rock and concrete materials in this model, and the distributive parameters can be easily determined. This model is applicable to the brittle materials without large plastic strain or plastic slipping. It may not be suitable to describe soft rock or deep-bedded rock with the feature of plastic flow, according to the basic assumptions of the model. Further work may consider size effect, characteristic scale of the fracture and energy dissipation of the failure process.

\section{References}

[1] Jing,L., A review of techniques, advances and outstanding issues in numerical modeling for rock mechanics and rock engineering. International Journal of Rock Mechanics and Mining Sciences, 2003, 40(3): 283-353.

[2] Yue,Z.Q., et al., Finite element modeling of geo materials using digital image processing. Computers and Geotechnics, 2003, 30(5): 375-397.

[3] Wang,X.B., Stain localization and complete stress-strain curve of rock specimen in uniaxial tension. Chinese Journal of Rock Mechanics and Engineering, 2005, 24(Supp2): 5784-5788. 
[4] Liu,X.L. and Wen,B., Strain-softening behavior and description of concrete in uniaxial tension. Engineering Mechanics, 1998, A01: 8-18.

[5] Hughes,B.P. and Gregory,R., Concrete subjected to high rates of loading in compression. Magazine of Concrete Research, 1972, 24(78): 25-36.

[6] Popovers,S., A review of stress-strain relationships for concrete. ACI Journal Proceedings, ACI, 1970, 67(3): 243-248.

[7] Guo,Z.H. and Zhang,X.Q., Experimentally study on complete stress-strain curve of concrete in uniaxial tension. Journal of building structures, 1988, 4: 45253.

[8] Cao,W.G., et al., A study of statistical constitutive model for soft and damage rocks. Chinese Journal of Rock Mechanics and Engineering, 1998, 17(6): 628-633.

[9] Xu,W.Y. and Wei,L.D., Study on statistical damage constitutive model of rock. Chinese Journal of Rock Mechanics and Engineering, 2002, 21(6): 787-791.

[10] Shen,Z.J. and Chen,T.L., Breakage mechanic of geomaterial-structure types and load sharing. Chinese Journal of Rock Mechanics and Engineering, 2004, 23(13): 2137-2142.

[11] Li,S.H. and Zhou,D., Formulation for damage of brittle materials and computational element based on criterion of strain strength distribution. Journal of Hydraulic Engineering, 2012, 43(s1): 8-12.

[12] Li,S.H. and Zhou,D., Computation model of progressive failure plane in geological body and failure criterion of shear plan. Chinese Journal of Rock Mechanics and Engineering, 2013, z2: 3009-3015.

[13] Li,S.H. and Zhou,D., Progressive failure constitutive model of fracture plane in geometrical based on strain strength distribution, International Journal of Solids and Structures, 2013, 50(3-4): 570-577.

[14] Ansari,F., Stress-strain response of microcracked concrete in direct tension. ACI Materials Journal, 1987, 84(6): 481-490.

[15] Wu,F., Experimental Study on Whole Stress-strain Curves of Concrete Under Axial Tension. Changsha: Hunan University College of Civil Engineering, 2006.

[16] Lawson, et al., Solving Least Squares Problems. Englewood Cliffs, NJ: Prentice-hall, 1974, 161.

[17] Bates, et al., Nonlinear Regression: Iterative Estimation and Linear Approximations. John Wiley \& Sons, Inc., 1988.

[18] Dennis,J., et al., Numerical methods for unconstrained optimization and nonlinear equations. Siam, 1996, 16.

[19] Okubo,S. and Fukui,K., Complete stress-strain curves for various rock types in uniaxial tension. International Jourmal of Rock Mechanics and Mining Sciences \& Geomechanics Abstracts, Pergamon, 1996, 33(6): 549-556. 